\title{
Vitesse de croissance dans le lait de Listeria monocytogenes et autres souches du même genre à des températures suboptimales
}

\author{
HP Siswanto, J Richard
}

\author{
INRA, Station de recherches laitières, 78350 Jouy-en-Josas, France
}

(Reçu le 2 septembre 1991; accepté le 25 février 1992)

\begin{abstract}
Résumé - À partir des cultures de 3 souches de référence de Listeria monocytogenes sur du lait écrémé à 6 températures comprises entre 4 et $35^{\circ} \mathrm{C}$, on a montré que le modèle simplifié de Ratkowsky et al (1982), reliant la vitesse de croissance des microorganismes à la température de culture, était applicable sur cette gamme de températures. En effet, on observait une relation linéaire entre la racine carrée du taux de croissance et la température de culture de ces bactéries. On a déterminé ensuite les temps de génération à 8,30 et $35^{\circ} \mathrm{C}$ de 16 souches de L monocytogenes, dont les 3 précédentes, et de 6 souches appartenant à d'autres espèces du genre Listeria. À l'aide du modèle simplifié et des résultats obtenus à 2 températures seulement ( 8 et $30^{\circ} \mathrm{C}$ ), on a fait ensuite une prédiction du taux de croissance de ces bactéries à 3 températures d'intérêt technologique : 4,12 et $35^{\circ} \mathrm{C}$. Dans l'ensemble, on n'a pas pu mettre en évidence de différence significative de vitesse de croissance entre les souches de $L$ monocytogenes et les souches des autres espèces de Listeria, ni entre les sérotypes 1 et 4 de $L$ monocytogenes. Le modèle simplifié permet de prédire que la conservation du lait à $4{ }^{\circ} \mathrm{C}$, pendant une durée inférieure à $3 \mathrm{j}$, ne devrait pas autoriser la croissance de Listeria monocytogenes. $\grave{A} 12^{\circ} \mathrm{C}$, température de maturation du lait destiné à la fabrication de certains fromages, le temps prédit de génération des Listeria est compris entre 5 et $6 \mathrm{~h}$. On pourrait donc avoir de 3 à 4 générations en $18 \mathrm{~h}$, si la période de maturation du lait était aussi longue. Le calcul permet de prévoir également de 3 à 4 générations durant la fabrication de fromage à pâte molle (température s'abaissant de 35 à $25^{\circ} \mathrm{C}$ ), dans l'hypothèse de l'absence de compétition avec la flore lactique dans les 3 à 4 premières $h$ de développement de cette flore. $\dot{A} 35{ }^{\circ} \mathrm{C}$, température maximale de fabrication de nombreux fromages, les temps de génération calculés étaient en général supérieurs à ceux réellement observés. La validité du modèle simplifié n'est donc pas assurée pour toutes les souches de Listeria au-delà de $30^{\circ} \mathrm{C}$.
\end{abstract}

Listeria / croissance / lait / température / modélisation

Summary - Growth rate of Listeria monocytogenes and other species of Listeria in the milk at sub-optimum temperatures. The simplified model of Ratkowsky et al (1982) to predict the growth rate of Listeria monocytogenes as a function of temperature was validated by culturing 3 reference strains (V7, Scott $A$ and California) in skim-milk at 6 different temperatures ranging from $4-35{ }^{\circ} \mathrm{C}$. Then the relationship between the temperature and the growth rate of 13 strains of $\mathrm{L}$ monocytogenes (7 serotype 1 and 6 serotype 4) including the reference ones and 6 other species of Listeria was determined using only 2 cultivation temperatures $\left(8\right.$ and $\left.30^{\circ} \mathrm{C}\right)$. This relation was used to calculate the bacterial growth rates at 3 temperatures of technological interest: 4,12 and $35^{\circ} \mathrm{C}$. On 
average, there was no significant difference in generation times between the strains of $\mathrm{L}$ monocytogenes and those belonging to the other species of Listeria or between L monocytogenes serotypes 1 and 4. At $4{ }^{\circ} \mathrm{C}$, the temperature of milk storage, generation times of $\mathrm{L}$ monocytogenes were slightly higher than those of the other Listeria strains. Moreover, it can be predicted they will not be able to grow in milk at $4{ }^{\circ} \mathrm{C}$ during the first 3 days of storage. At $12{ }^{\circ} \mathrm{C}$, the temperature of milk ripening before certain traditional cheese making, the Listeria generation times were between 5-6 h. Consequently, they could have 3-4 generations within $18 \mathrm{~h}$, which is a very long milk ripening period. This calculation also allows prediction of 3 to 4 generations during the manufacture of soft-cheese (temperature drop from 35 to $25^{\circ} \mathrm{C}$ ), if there is no competition with the lactic flora during the first $3-4 \mathrm{~h}$ of milk fermentation. At $35{ }^{\circ} \mathrm{C}$, the maximum temperature for manufacture of some cheeses, the observed generation times were generally lower than those calculated. Consequently, the validity of the simplified model does not hold for all Listeria strains cultivated in milk at temperatures over $30^{\circ} \mathrm{C}$.

\section{Listeria / growth / milk / temperature / modelisation}

\section{INTRODUCTION}

La nature psychrotrophe de Listeria monocytogenes est bien établie (Wilkins et al, 1972; Hayes et al, 1986; Donnelly et Briggs, 1986; Junttila et al, 1988). Par exemple, on a observé que Listeria monocytogenes peut croître à $4{ }^{\circ} \mathrm{C}$ dans le lait pasteurisé (Donnelly et Briggs, 1986; Rosenow et Marth, 1987), à $3^{\circ} \mathrm{C}$ sur gélose Tryptose Phosphate (Hof et al, 1986), à $0{ }^{\circ} \mathrm{C}$ dans la viande stérile (Khan et al, 1972 ) et même à une température aussi basse que $-0,4^{\circ} \mathrm{C}$ dans du lait UHT (Walker et al, 1990). Listeria monocytogenes peut aussi se multiplier à basse température dans les fromages, si le $\mathrm{pH}$ n'y est pas trop bas (Ryser et al, 1985; Ryser et Marth, 1987a, 1987b; Yousef et Marth, 1988). Par conséquent, la conservation au froid de beaucoup de produits alimentaires n'entrave pas la croissance de Listeria monocytogenes (Marshall et Schmidt, 1988).

D'un point de vue pratique cependant, il est intéressant de connaître la vitesse de croissance de ces bactéries dans une gamme de températures qui peuvent être rencontrées au cours de la fabrication et de la conservation des produits alimentaires. En technologie fromagère, il s'agit en particulier de la température de conser- vation du lait cru à la ferme, et de la fabrication des fromages avant que le caillé ne devienne suffisamment acide pour inhiber les Listeria. C'est-à-dire, en général, une gamme de températures variant de 4 à $35^{\circ} \mathrm{C}$.

Rosenow et Marth (1987) ont déterminé les temps de génération dans le lait de 4 souches de référence de $L$ monocytogenes, à des températures comprises entre 4 et $21^{\circ} \mathrm{C}$ pour 3 d'entre elles, et jusqu'à $35^{\circ} \mathrm{C}$ pour la $4^{e}$. II est donc difficile de tirer des conclusions, applicables à l'ensemble des souches de cette espèce, d'une étude aussi limitée.

Cependant, l'élargissement de ce type d'investigation à d'autres souches représenterait un travail considérable, s'il était réalisé dans les mêmes conditions que celles utilisées par Rosenow et Marth. Le travail se trouve simplifié depuis que Ratkowsky et al (1982) ont montré l'existence, à température inférieure à l'optimum de croissance de nombreuses bactéries, d'une relation linéaire entre la racine carrée du taux de croissance et la température de culture de ces bactéries (modèle simplifié). En effet, l'estimation du taux de croissance de celles-ci à 2 températures convenablement choisies devrait suffire pour établir cette relation et par la suite, pour déterminer leur taux de croissance à 
toute température sub-optimale. II devrait donc être possible, sans trop de travail, de comparer la vitesse de croissance d'une collection de souches sur la base de 2 paramètres seulement : la relation «taux de croissance-température de culture" et le taux de croissance à une température de culture inférieure à l'optimum.

On a entrepris, dans notre laboratoire, une étude de l'influence de la température sur la durée de la phase de latence et sur le taux de croissance dans le lait de 13 souches de Listeria monocytogenes appartenant aux sérotypes 1 et 4 , et de 6 souches d'autres espèces du même genre. Les résultats obtenus par Rosenow et Marth (1987) ont d'abord été complétés puis analysés, de façon à voir dans quelle mesure le modèle simplifié proposé par
Ratkowsky et al (1982) était applicable à la croissance de Listeria monocytogenes dans le lait, dans la gamme de température allant de 4 à $35^{\circ} \mathrm{C}$.

\section{MATÉRIELS ET MÉTHODES}

\section{Origine et conservation des souches}

L'origine des souches utilisées dans cette étude est indiquée dans le tableau I. Les 3 souches de $L$ monocytogenes utilisées dans l'étude de Rosenow et Marth (1987) ont été reprises comme référence pour une investigation préliminaire : V7, Scott A (SA) et California (CA). Par la suite, on a utilisé 10 autres souches de $L$ monocytogenes, dont 6 de sérotype 1 et 4 de sérotype 4 , et 6 souches de Listeria d'autres espèces :

Tableau I. Nature et origine des espèces de Listeria utilisées.

Nature and origin of the strains of Listeria.

\begin{tabular}{llll}
\hline Code & Espèce & Sérotype & Origine \\
& & & \\
V7 & L monocytogenes & 1 & lait cru \\
SA & L monocytogenes & 4 & Scott A, isolat clinique \\
CA & L monocytogenes & 4 & fromage, épidémie de Californie \\
LM 1 & L monocytogenes & 1 & lait \\
LM 2 & L monocytogenes & 1 & lait \\
LM 3 & L monocytogenes & 1 & lait cru \\
LM 4 & L monocytogenes & 1 & lait \\
LM 5 & L monocytogenes & 1 & fromage \\
LM 6 & L monocytogenes & 1 & fromage \\
LM 7 & L monocytogenes & 4 & lait \\
LM 8 & L monocytogenes & 4 & fromage \\
LM 9 & L monocytogenes & 4 & pate molle (soft cheese) \\
LM 10 & L monocytogenes & 4 & lait \\
LM 1 & L innocua & $6 \mathrm{~A}$ & environnement industriel viande \\
LIN 2 & L innocua & $6 \mathrm{~B}$ & fromage \\
LIV & L ivanovii & 5 & environnement laitier \\
LSE 1 & L seeligeri & $6 \mathrm{~B}$ & viande \\
LSE 2 & L seeligeri & $1-2$ & fromage \\
LWE & L welshimeri & nd & environnement laitier \\
& & & \\
\hline
\end{tabular}

Souches LM 1 à LM 10 : gracieusement fournies par le Pr Audurier, hôpital de la faculté de médecine de Tours: souches V7, SA et CA : Dr E Ryser (University of Wisconsin); autre souches : en provenance d'un laboratoire privé. Strains LM1 to LM10: kindly supplied by Prof Audurier, CHU de Tours; strains V7, SA and CA: Dr E Ryser (University of Wisconsin); other strains: obtained from a private laboratory. 
L welshimeri (1 souche), L innocua (2 souches), $L$ seeligeri (2 souches) et L ivanovii (1 souche).

Les cultures étaient conservées à $4^{\circ} \mathrm{C}$ sur gélose Tryptose (Difco 64) et transférées chaque mois.

Pour préparer une culture active pour chaque expérimentation, les souches stockées étaient transférées sur bouillon Tryptose (Difco, 62 ) et incubées $24 \mathrm{~h}$ à $30^{\circ} \mathrm{C}$ en aérobiose. Une première culture sur lait était réalisée en ajoutant $1 \mathrm{ml}$ de la culture sur bouillon à $100 \mathrm{ml}$ de lait écrémé reconstitué $(10 \%$ de poudre de I'Union laitière normande (ULN, 50890 Condésur-Vire, France) garantie sans antibiotique), stérilisé à $110^{\circ} \mathrm{C}$ pendant $10 \mathrm{~min}$. Après $24 \mathrm{~h}$ d'incubation à $30^{\circ} \mathrm{C}$, les bactéries étaient arrivées en phase stationnaire de croissance, à une population maximale d'environ $1,6 \times 10^{8}$ ufc/ml.

Une dilution de cette préculture dans le même lait était effectuée de façon à obtenir une population initiale de $1,2 \times 10^{5}$ à $3,3 \times 10^{5} \mathrm{ufc} /$ $\mathrm{ml}$.

\section{Conditions de culture}

Des volumes de $100 \mathrm{ml}$ du lait écrémé ainsi inoculé étaient placés dans des flacons de $250 \mathrm{ml}$ et incubés au bain-marie à 21,30 et $35^{\circ} \mathrm{C}$. Pour la culture à plus basse température $\left(8^{\circ} \mathrm{C}\right)$, le lait était réparti en tubes de $5 \mathrm{ml}$, puis mis à incuber dans un bain-marie installé dans une chambre froide.

Pour les cultures incubées à 21,30 et $35^{\circ} \mathrm{C}$, des échantillons étaient prélevés chaque heure pendant $10 \mathrm{~h}$, et puis après 14 et $24 \mathrm{~h}$ d'incubation. Pour celles incubées à plus basse température, les prélèvements étaient effectués tous les 2 j, jusqu'au $20^{\ominus} \mathrm{j}$ inclus.

\section{Dénombrement des Listeria}

Le lait était agité modérément avant prélèvement pour l'homogénéiser, tout en évitant une forte aération du milieu. La prise d'essai était diluée dans de l'eau peptonée à $0,1 \%$, avant ensemencement à la surface de la gélose TSA.YE (milieu "Tryptic Soy Agar", Difco 369 additionné de $0,6 \%$ d'extrait de levure, Diagnostics Pas- teur). Cet ensemencement était effectué à l'aide d'un ensemenceur Spiral (Spiral System, Model DS). Les colonies étaient comptées après $24 \mathrm{~h}$ d'incubation à $30^{\circ} \mathrm{C}$.

Les courbes de croissance étaient tracées pour chaque souche sous forme du $\log _{10} d u$ nombre d'unitès formant colonies (log ufc/ml) en fonction du temps. La phase exponentielle de croissance était délimitée visuellement et le calcul de la pente $p$ (log ufc $\left.\mathrm{ml}^{-1} \cdot \mathrm{h}^{-1}\right)$ durant cette phase linéaire de croissance était effectué par régression à partir des points apparemment alignés. Le logiciel utilisé (Statiff, Institut technique des céréales de France, Paris) donnait aussi l'écart-type $s_{b}$ de cette estimation, à partir duquel on pouvait calculer l'intervalle de confiance de la valeur de la pente (Snedecor et Cochran, 1957).

\section{Calcul des taux de croissance}

Les taux de croissance $\mu\left(\mathrm{h}^{-1}\right)$ étaient calculés à l'aide de la formule suivante :

$$
\mu=p \times 3,32
$$

où $p$ est la pente obtenue précédemment, et 3,32 le terme correctif pour passer des logarithmes de base 10 à ceux de base 2. Le temps de génération (en h) est obtenu en calculant l'inverse du taux de croissance.

\section{Modélisation de la relation "taux de croissance-température de culture"}

On a utilisé le modèle simplifié proposé par Ratkowsky et al $(1982,1983)$ pour relier la vitesse de croissance à une température de culture sub-optimale :

$$
\sqrt{\mu}=b\left(T-T_{m}\right)
$$

où $\mu$ est le taux de croissance, $b$ la pente de la régression linéaire, $T$ la température de culture réelle (en kelvins) et $T_{\mathrm{m}}$ la température de culture minimale (théorique), obtenue en donnant à $\mu$ la valeur 0 dans la relation (1).

Dans l'étude préliminaire, portant sur 3 souches de $L$ monocytogenes et 6 températures de culture, la racine carrée des taux de crois- 
sance était représentée en fonction de la température de culture exprimée en degrés Celsius, ce qui revient simplement à faire un changement d'échelle, La pente de la partie linéaire de la courbe obtenue était calculée par régression linéaire, à l'aide du logiciel Statitcf.

Pour l'ensemble des souches de la collection (étude générale), les cultures étaient effectuées à 3 températures seulement : 8,30 et $35^{\circ} \mathrm{C}$. Pour chaque souche, le paramètre $b$, établissant la relation entre le taux de croissance et la température de culture, était alors calculée de la façon suivante :

$$
b=\frac{\sqrt{\mu_{2}}-\sqrt{\mu_{1}}}{\Theta_{2}-\Theta_{1}}
$$

où $\mu_{1}$ et $\mu_{2}$ sont les taux de croissance à respectivement $8^{\circ} \mathrm{C}\left(\Theta_{1}\right)$ et à $30^{\circ} \mathrm{C}\left(\Theta_{2}\right)$.

\section{RÉSULTATS}

\section{Étude préliminaire (validité du modèle simplifié de Ratkowsky et al, 1982)}

La figure 1 donne un exemple de courbe de croissance de Listeria monocytogenes (souche Scott A) dans le lait écrémé à $35^{\circ} \mathrm{C}$. Sur cette courbe, on remarque une phase de latence et d'accélération d'environ $1 \mathrm{~h}$, puis une portion linéaire de la $1^{\text {re }}$ jusqu'à la $8^{\mathrm{e}} \mathrm{h}$, et enfin une phase de ralentissement de $2 \mathrm{~h}$, suivie d'une phase stationnaire, à partir de $10 \mathrm{~h}$ de culture. Après $14 \mathrm{~h}$, la population maximale de cette bactérie restait stable à environ $1,6 \mathrm{x}$ $10^{8} \mathrm{ufc} / \mathrm{ml}$. L'analyse de régression de la portion linéaire de la courbe de croissance cellulaire donne une pente $p=0,35 \pm 0,03$ (unité de log ufc. $\mathrm{ml}^{-1} \cdot \mathrm{h}^{-1}$ ). On en déduit un taux de croissance égal $1,16 \mathrm{~h}^{-1}$, soit un temps de génération de $0,86 \mathrm{~h}$ (environ 52 $\min )$.

Les taux de croissance obtenus à 6 autres températures entre 4 et $35^{\circ} \mathrm{C}$, et pour les 3 souches (V7, SA et CA), ont été

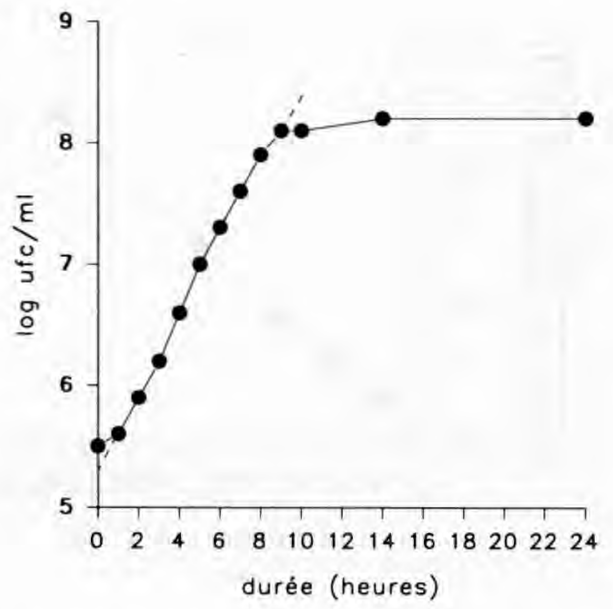

Fig 1. Croissance de Listeria monocytogenes (souche Scott A) à $35^{\circ} \mathrm{C}$ dans du lait écrémé. Pente au cours de la phase de croissance exponentielle (de 1 à $8 \mathrm{~h}$ ) : $3,35 \pm 0,03 \log _{10}$ ufc. $\mathrm{ml}^{-1}$. $\mathrm{h}^{-1}$.

Growth of Listeria monocytogenes (strain Scott $A$ ) in skim milk at $35^{\circ} \mathrm{C}$. Slopes during the exponential growth phase $(1-8 \mathrm{~h}): 3.35 \pm 0.03 \log _{10}$ cfu. $m r^{-1} \cdot h^{-1}$.

déterminés de la même façon, puis une représentation graphique de la relation racine carrée du taux de croissance en fonction de la température a été réalisée. La figure 2 illustre cette représentation. On observe que les valeurs $\sqrt{\mu}$ obtenues à partir de nos résultats sont très voisines de celles de Rosenow et Marth (1987) et sont également bien alignées. Cela signifie que le modèle simplifié de Ratkowsky et al (1982) peut être utilisé pour présenter la relation entre le taux de croissance et la température de culture sur lait de $L$ monocytogenes, au moins dans cette gamme de température (de 4 à $35^{\circ} \mathrm{C}$ ), et pour les 3 souches. L'intersection de la droite de régression avec l'axe des abscisses permet de déterminer la température minimale théorique de croissance de la souche, 

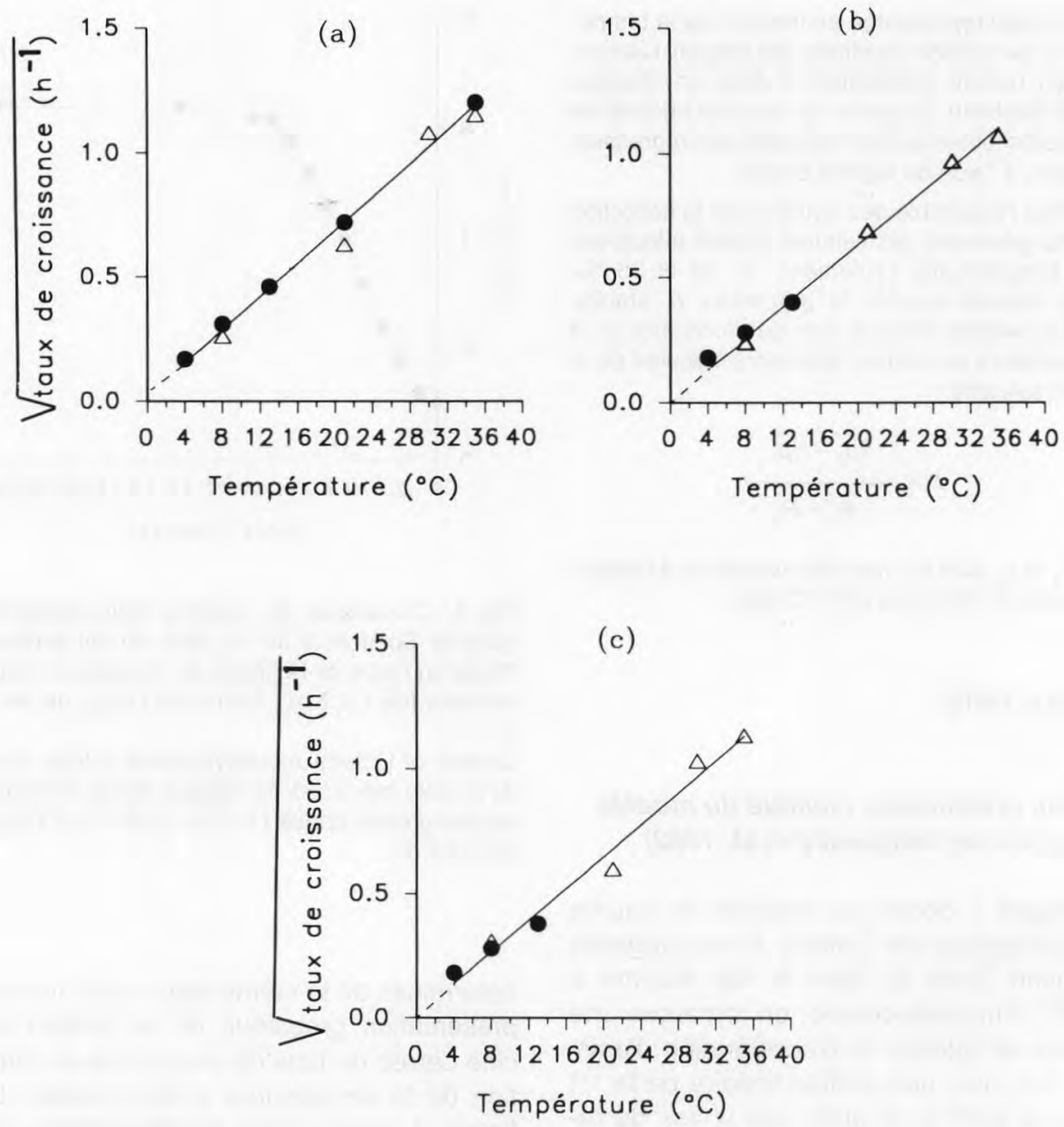

Fig 2. Représentation, par le modèle simplifié de Ratkowsky et al (1982), de la relation entre le taux de croissance de Listeria monocytogenes et la température de culture de cette bactérie dans du lait écrémé. (a), (b) et (c) : Souches V7, Scott A et California, respectivement; - résultats obtenus par Rosenow et Marth (1987); $\Delta$ résultats de la présente étude.

Relationship between the growth rate of Listeria monocytogenes in skim milk and the temperature of cultivation, using the simplified model of Ratkowsky et al (1982). (a), (b) et (c): Strain V7, Scott A and California, respectively. : results obtained by Rosenow and Marth (1987); $\Delta$ : results of this study.

dans l'hypothèse (rarement vérifiée) où le modèle serait linéaire jusque-là.

Le tableau II rassemble les paramètres de croissance fournis par le modèle, pour les 3 souches de références de $L$ monocy- togenes. Pour la souche V7, on n'observe pas de différence significative entre la valeur du paramètre $b$ obtenue à partir des résultats de Rosenow et Marth (1987) et celle basée sur nos propres résultats. 
Tableau II. Paramètres de croissance de $L$ monocytogenes dans le lait écrémé en fonction de la température de culture.

Growth parameters of $\mathrm{L}$ monocytogenes in skim milk as a function of culture temperature.

\begin{tabular}{rrrrrr}
\hline Souche & NTC & $\begin{array}{c}\text { Intervalle } \\
\text { de température }\left({ }^{\circ} \mathrm{C} \text { ) }\right.\end{array}$ & Paramètre b & $\begin{array}{c}\text { Temps de } \\
\text { génération à } 0{ }^{\circ} \mathrm{C} \text { (jour) }\end{array}$ & $\begin{array}{c}\text { Température } \\
\text { minimale }\left({ }^{\circ} \mathrm{C} \text { ) }\right.\end{array}$ \\
\hline $\mathrm{V} 7$ & $\mathrm{R}: 5$ & $4-35$ & $0,033 \pm 0,001$ & 32 & $-1,1$ \\
& $\mathrm{~S}: 4$ & $8-35$ & $0,035 \pm 0,014$ & 32 & $+1,0$ \\
& $\mathrm{R}+\mathrm{S}: 9$ & $4-35$ & $0,033 \pm 0,003$ & 120 & $-0,6$ \\
$\mathrm{SA}$ & $\mathrm{R}: 3$ & $4-13$ & $0,022 \pm 0,15$ & 3 & $-5,0$ \\
& $\mathrm{~S}: 4$ & $8-35$ & $0,031 \pm 0,008$ & 720 & $-0,2$ \\
& $\mathrm{R}+\mathrm{S}: 7$ & $4-35$ & $0,030 \pm 0,003$ & 45 & $-1,0$ \\
$\mathrm{CA}$ & $\mathrm{R}: 3$ & $4-13$ & $0,022 \pm 0,018$ & 5 & $-4,3$ \\
& $\mathrm{~S}: 4$ & $8-35$ & $0,032 \pm 0,016$ & 100 & $-0,6$ \\
& $\mathrm{R}+\mathrm{S}: 7$ & $4-35$ & $0,032 \pm 0,005$ & 77 & $+0,7$ \\
\hline
\end{tabular}

NTC : nombre de températures différentes de culture; R : étude de Rosenow et Marth (1987); S : présente étude; paramètre $b$ : valeur de la pente $\left(\sqrt{\mu} .{ }^{\circ} \mathrm{C}^{-1}\right)$ dans le modèle de Ratkowsky et al (1982), avec intervalle de confiance à $P=0,95$.

Cette dernière est cependant estimée avec moins de précision. Les températures minimales de croissance sont très proches.

Pour les souches SA et CA, les valeurs du paramètre $b$ calculées à partir des taux de croissance obtenus par Rosenow et Marth (1987) sont nettement plus faibles que celles dérivées de nos propres résultats. La différence n'est cependant pas significative, du fait d'intervalles de confiance larges. Par ailleurs, les températures minimales calculées à partir des résultats de Rosenow et Marth sont invraisemblablement basses. Ces divergences résultent essentiellement de la différence d'intervalles de température explorés dans ces deux études, celui de Rosenow et Marth étant plus réduit que le nôtre. En regroupant les résultats de ces deux études, nous obtenons des valeurs du paramètre $b$ plus précises, et des températures minimales plus vraisemblables. On observe alors que les 3 souches ne présentent pas de différence significative sur le plan de la relation entre le taux de croissance et la température.

\section{Étude avec les autres souches}

Le tableau III présente, pour l'ensemble des souches de Listeria, les temps de génération à 8,30 et $35^{\circ} \mathrm{C}$ réellement observés, et les temps de génération à 4,12 et $35{ }^{\circ} \mathrm{C}$, calculés en utilisant le modèle de Ratkowsky et les taux de croissance à 8 et $30{ }^{\circ} \mathrm{C}$ seulement. La relation entre le taux de croissance et la température de culture (paramètre $b$ ) est également présentée.

Compte tenu des variations entre souches, on n'observe pas de différence significative de temps réels de génération à 8,30 et $35^{\circ} \mathrm{C}$ entre les différents groupes de souches. À $8^{\circ} \mathrm{C}$ par exemple, on $\mathrm{a}$, dans chaque groupe de souches, 
Tableau III. Paramètres de croissance de différentes espèces et souches de Listeria dans le lait écrémé.

Growth parameters of different strains of Listeria in skim milk as a function of culture temperature.

\begin{tabular}{|c|c|c|c|c|c|c|c|}
\hline \multirow[t]{3}{*}{ Souche } & \multicolumn{6}{|c|}{ Temps de génération (h) } & \multirow[t]{3}{*}{ Paramètre $\mathrm{b}$} \\
\hline & \multicolumn{3}{|c|}{ observés } & \multicolumn{3}{|c|}{ calculés } & \\
\hline & $8^{\circ} \mathrm{C}$ & $30^{\circ} \mathrm{C}$ & $35^{\circ} \mathrm{C}$ & $4^{\circ} \mathrm{C}$ & $12^{\circ} \mathrm{C}$ & $35^{\circ} \mathrm{C}$ & \\
\hline $\begin{array}{l}\text { V7 } \\
\text { SA } \\
\text { CA }\end{array}$ & $\begin{array}{l}12,0 \\
14,3 \\
11,1\end{array}$ & $\begin{array}{l}0,9 \\
1,1 \\
0,9\end{array}$ & $\begin{array}{l}0,7 \\
0,9 \\
0,8\end{array}$ & $\begin{array}{l}30,9 \\
49,6 \\
35,4\end{array}$ & $\begin{array}{l}6,1 \\
5,7 \\
5,6\end{array}$ & $\begin{array}{l}0,9 \\
0,8 \\
1,0\end{array}$ & $\begin{array}{l}0,034 \\
0,032 \\
0,033\end{array}$ \\
\hline Moyenne & 12,5 & 1,0 & 0,8 & 38,6 & 5,8 & 0,9 & 0,033 \\
\hline $\begin{array}{l}\text { LM } 1 \\
\text { LM } 2 \\
\text { LM } 3 \\
\text { LM } 4 \\
\text { LM } 5 \\
\text { LM } 6\end{array}$ & $\begin{array}{l}13,1 \\
12,0 \\
11,1 \\
14,0 \\
11,1 \\
12,0\end{array}$ & $\begin{array}{l}0,8 \\
0,7 \\
1,1 \\
1,4 \\
1,0 \\
0,8\end{array}$ & $\begin{array}{l}0,8 \\
0,8 \\
0,8 \\
0,9 \\
0,9 \\
0,9\end{array}$ & $\begin{array}{l}63,0 \\
65,0 \\
30,9 \\
27,7 \\
35,4 \\
42,2\end{array}$ & $\begin{array}{l}5,2 \\
4,9 \\
5,7 \\
6,6 \\
5,6 \\
5,1\end{array}$ & $\begin{array}{l}0,6 \\
0,5 \\
0,8 \\
1,2 \\
0,8 \\
0,6\end{array}$ & $\begin{array}{l}0,039 \\
0,041 \\
0,030 \\
0,025 \\
0,032 \\
0,036\end{array}$ \\
\hline Moyenne & 12,2 & 1,0 & 0,8 & 44,0 & 5,5 & 0,8 & 0,034 \\
\hline $\begin{array}{l}\text { LM } 7 \\
\text { LM } 8 \\
\text { LM } 9 \\
\text { LM } 10\end{array}$ & $\begin{array}{l}12,0 \\
12,7 \\
12,7 \\
14,0\end{array}$ & $\begin{array}{l}0,9 \\
0,9 \\
1,0 \\
0,9\end{array}$ & $\begin{array}{l}0,9 \\
0,9 \\
0,8 \\
0,8\end{array}$ & $\begin{array}{l}39,1 \\
41,1 \\
43,3 \\
55,7\end{array}$ & $\begin{array}{l}5,2 \\
5,5 \\
5,8 \\
5,6\end{array}$ & $\begin{array}{l}0,6 \\
0,7 \\
0,8 \\
0,6\end{array}$ & $\begin{array}{l}0,035 \\
0,034 \\
0,033 \\
0,036\end{array}$ \\
\hline Moyenne & 12,9 & 0,9 & 0,9 & 44,8 & 5,5 & 0,7 & 0,035 \\
\hline $\begin{array}{l}\text { LWE } \\
\text { LIN } 1 \\
\text { LIN } 2 \\
\text { LSE 1 } \\
\text { LSE 2 } \\
\text { LIV }\end{array}$ & $\begin{array}{l}11,6 \\
12,7 \\
14,0 \\
11,6 \\
12,7 \\
14,0\end{array}$ & $\begin{array}{l}1,0 \\
1,2 \\
0,9 \\
0,9 \\
1,0 \\
1,2\end{array}$ & $\begin{array}{l}0,9 \\
0,8 \\
0,9 \\
0,8 \\
0,8 \\
0,9\end{array}$ & $\begin{array}{l}33,0 \\
21,4 \\
55,7 \\
39,1 \\
43,3 \\
21,4\end{array}$ & $\begin{array}{l}5,6 \\
5,0 \\
5,6 \\
5,2 \\
5,8 \\
5,0\end{array}$ & $\begin{array}{l}0,8 \\
0,7 \\
0,9 \\
0,7 \\
0,6 \\
0,9\end{array}$ & $\begin{array}{l}0,031 \\
0,029 \\
0,036 \\
0,035 \\
0,033 \\
0,029\end{array}$ \\
\hline Moyenne & 12,8 & 1,0 & 0,8 & 35,7 & 5,4 & 0,8 & 0,032 \\
\hline
\end{tabular}

$b=\sqrt{\mu_{2}}-\sqrt{\mu_{1}} / \Theta_{2}-\Theta_{1}\left(\mathbf{b}\right.$, pente dans le modèle de Ratkowsky et al, 1982) où $\mu_{1}$ et $\mu_{2}$ sont les taux de croissance à respectivement $8^{\circ} \mathrm{C}\left(\Theta_{1}\right)$ et $30^{\circ} \mathrm{C}\left(\Theta_{2}\right)$.

des temps de génération variant de 11 à $14 \mathrm{~h}$ environ (soit une amplitude de $3 \mathrm{~h}$ ).

Pour les 3 souches de référence (V7, $\mathrm{SA}$ et $\mathrm{CA}$ ), les valeurs du paramètre $b$ calculées à partir des taux de croissance à 2 températures seulement sont très proches de celles que l'on a obtenues précédemment avec 6 températures de culture : les écarts sont au plus de 2 centièmes d'unité, soit de l'ordre de $6 \%$. Avec les autres souches, on a des paramètres $b$ s'échelonnant entre 0,025 (souche LM4) et 0,041 
(souche LM2), sans qu'il apparaisse de différences significatives entre groupes. Au total, 14 souches sur 19 (soit de l'ordre de $74 \%$ ) sont à moins de 3 centièmes de la valeur moyenne générale du paramètre $b$, trouvée égale à $0,033 \pm 0,002$.

À partir des valeurs du paramètre $b$ et des taux réels de croissance à $30^{\circ} \mathrm{C}$, on a calculé les temps de génération de toutes les souches de Listeria à 4,12 et $35^{\circ} \mathrm{C}$.

A $4{ }^{\circ} \mathrm{C}$, la moyenne des temps estimés de génération des souches de $L$ monocytogenes est supérieure à celle des autres souches de Listeria ( $44 \mathrm{~h}$ contre $35 \mathrm{~h}$ ) avec, cependant, de très grandes variations à l'intérieur de chaque groupe, de sorte que ces différences ne sont pas significatives. Ces variations peuvent atteindre $35 \mathrm{~h}$ dans un même groupe (par exemple, groupe de $L$ monocytogenes, sérotype 1, souches $L M 1$ à LM6), c'est-à-dire nettement plus qu'avec les cultures à $8^{\circ} \mathrm{C}$.

A $12^{\circ} \mathrm{C}$, le temps calculé de génération de la plupart des Listeria est compris entre 5 et $6 \mathrm{~h}$, quel que soit le groupe de bactéries considéré. II est également remarquable que les différences entre souches soient aussi faibles : moins de $1,7 \mathrm{~h}$ entre le temps de génération le plus long (souche LM4) et le temps de génération le plus court (souche LM2).

À $35^{\circ} \mathrm{C}$, les temps de génération calculés varient de $0,5 \mathrm{~h}$ (souche LM2) à 1,2 h (souche LM4) sans qu'il apparaisse de différence entre groupes. L'amplitude de $0,7 \mathrm{~h}$ environ entre les valeurs extrêmes est nettement plus grande qu'avec les valeurs observées (amplitude de 0,2 h seulement). D'autre part, les temps de génération prédits pour les souches de référence sont assez différents de ceux effectivement observés : on note des écarts de 0,1 à $0,2 \mathrm{~h}$, soit de 15 à $30 \%$ de variation. Ces différences peuvent être attribuées aux calculs effectués : imprécision normale des estimations de $\sqrt{\mu}$ à partir du modèle, am- plifiée par la transformation "carré" puis “inverse", pour avoir le temps de génération. Malgré ces erreurs dans les estimations des temps de génération, on observe que les valeurs calculées sont souvent inférieures à celles que l'on a effectivement observées (différence moyenne significative au seuil $P=0,05$ ). Cela signifiie que la validité du modèle simplifié de Ratkowsky n'est pas assurée pour toutes les souches de Listeria au-delà de $30^{\circ} \mathrm{C}$.

\section{DISCUSSION}

La validité du modèle simplifié de Ratkowsky a été vérifiée pour des bactéries thermophiles, mésophiles, psychrotrophes ou psychrophiles appartenant à divers genres et espèces (Ratkowsky et al, 1982 et 1983). II n'est donc pas étonnant que le modèle s'applique aussi à des bactéries du genre Listeria. Nous avons montré, avec 3 souches de référence, que cette relation était valable pour une gamme de température comprise entre 4 et $35^{\circ} \mathrm{C}$. Pour les autres souches de la collection, nous avons postulé qu'une relation linéaire était assurée dans un intervalle de températures plus restreint, de 8 à $30^{\circ} \mathrm{C}$, et nous avons déterminé le paramètre $b$ à partir de ces 2 températures de culture seulement. II en résulte des estimations de $b$ moins précises qu'avec un intervalle plus grand et avec plus de déterminations des taux de croissance. En conséquence, les vitesses prédites de croissance à diverses températures seront elles-mêmes moins précises. Cependant, si l'on admet que l'erreur sur l'estimation des taux de croissance à 8 et $30{ }^{\circ} \mathrm{C}$ est normalement distribuée, la moyenne des paramètres $b$ ou des taux calculés de croissance pour un groupe de bactéries, sera une estimation sans biais de la valeur réelle. II est donc légitime de comparer un ensemble de souches à un autre, sur la base de la valeur moyenne du 
paramètre $b$ ou du taux de croissance à une température donnée.

On peut estimer raisonnable l'interpolation à $12{ }^{\circ} \mathrm{C}$ que nous avons faite. Par contre, rien ne prouve que le modèle soit linéaire de 4 à $35^{\circ} \mathrm{C}$ pour toutes les souches de la collection. II est vraisemblable que certaines d'entre elles se multiplient plus lentement que prévu par le modèle à basse ou à haute température. Par conséquent, les prédictions que nous pouvons faire seront surestimées. Cela ne présente cependant pas d'inconvénient sur le plan pratique, puisqu'il s'agit d'évaluer un risque, et que le calcul réalisé surévalue ce dernier.

Compte-tenu des variations entre souches d'un même groupe, et du faible nombre de souches d'espèces autres que $L$ monocytogenes, on n'a pas réussi à mettre en évidence de différence significative entre groupes de ces bactéries sur la base des valeurs moyennes du paramètre b ou du taux de croissance à des températures de cultures particulières. D'un point de vue pratique, le plus important à retenir est que le temps de génération des Listeria dans le lait conservé à $4^{\circ} \mathrm{C}$ peut varier d'environ $21 \mathrm{~h}$ (souches LIN 1 et LIV) à $65 \mathrm{~h}$ (souche LM2). Cela signifie qu'avec les souches les plus rapides, on n'aurait pas plus de 3,6 générations en $3 \mathrm{j}$ de conservation du lait à $4{ }^{\circ} \mathrm{C}$, en supposant une croissance immédiate de ces souches. Or, il est bien établi, et nous l'avons vérifié dans la présente étude, que l'abaissement de la température de croissance de bactéries s'accompagne toujours de l'apparition d'une phase de latence. Dans l'étude de Rosenow et Marth, le temps de latence à $4^{\circ} \mathrm{C}$ était de l'ordre de 4 à $5 \mathrm{j}$, pour des conditions de culture (en particulier état physiologique de l'inoculum) tout à fait comparables aux nôtres. On peut donc dire que la conservation du lait à $4{ }^{\circ} \mathrm{C}$ pendant moins de $3 \mathrm{j}$ ne devrait pas permettre une croissance des Listeria qu'il contient.
À $12^{\circ} \mathrm{C}$ par contre, le temps calculé de génération des Listeria est compris entre 5 et $6 \mathrm{~h}$. On peut donc avoir 3 à 4 générations en $18 \mathrm{~h}$, la période de maturation la plus longue. Si l'on tient pour négligeable la phase de latence à cette température (les élévations de température du lait durant sa conservation à la ferme et sa collecte tenant lieu de phase de latence), cela aboutit à une multiplication par 8 ou par $16 \mathrm{du}$ nombre de Listeria par $\mathrm{ml}$ de lait. Ce n'est plus négligeable, sachant que le lait mis en œuvre pour une fabrication de fromage à pâte molle ne devrait pas contenir plus de 0,001 Listeria par $\mathrm{ml}(1 / \mathrm{l})$, pour être assuré de l'absence de cette bactérie dans $25 \mathrm{~g}$ de fromage en fin d'affinage (Ryser, comm pers). Si l'on considère un temps de génération moyen de l'ordre de $1 \mathrm{~h}$ entre 35 et $25^{\circ} \mathrm{C}$, variation de température durant la fabrication de beaucoup de fromages, et en admettant que la phase de latence est négligeable, on pourrait avoir à nouveau 3 à 4 générations de ces bactéries avant que l'acidification du lait n'entrave leur croissance. II est évident que pour éviter que cette multiplication affecte la qualité hygiénique des fromages, le niveau des Listeria à ce stade de fabrication doit être extrêmement bas. Une autre possibilité consiste à faire appel à des souches de bactéries lactiques productrices d'une substance inhibitrice vis-à-vis des Listeria (Maisnier-Patin et al, 1992).

\section{RÉFÉRENCES}

Donnelly CW, Briggs EH (1986) Psychrotrophic growth and thermal inactivation of Listeria monocytogenes as a function of milk composition. J Food Prot 49, 994-998, 1002

Hayes PS, Feeley JC, Graves LM, Ajello GW, Fleming DW (1986) Isolation of Listeria monocytogenes from raw milk. Appl Environ Microbiol $51,438-440$

Hof H, Seeliger HPR, Schrettenbrunner A, Chatzipanagiotou $S$ (1986) The role of $L$ monocytogenes and other Listeria spp in foodborne 
infection. In: Proc 2nd World Congr, Foodborne Infection and Intoxication. Berlin, 220223

Junttila JR, Niemelä SI, Hirn J (1988) Minimum growth temperatures of Listeria monocytogenes and non-haemolytic Listeria. J Appl Bacteriol 65, 321-327

Khan MA, Palmas CV, Seaman A, Woodbine M (1972) Survival versus growth of a facultative psychrotroph. Acta Microbiol Hung 19, 357362

Maisnier-Patin S, Deschamps N, Tatini SR, Richard J (1992) Inhibition of Listeria monocytogenes in Camembert cheese made with a nisin-producing starter. Lait 72, 249-263

Marshall DL, Schmidt RH (1988) Growth of Listeria monocytogenes at $10^{\circ} \mathrm{C}$ in milk preincubated with selected Pseudomonads. J Food Prot 51, 277-282

Ratkowsky DA, Olley J, McMekkin TA, Ball A (1982) Relationship between temperature and growth rate of bacterial cultures. J Bacteriol 149, 1-5

Ratkowsky DA, Lowry RK, McMeekin TA, Stockes AN, Chandler RE (1983) Model for bacterial culture growth rate throughout the entire biokinetic temperature range. J Bacteriol 154, 1222-1226

Rosenow EM, Marth EH (1987) Growth of Listeria monocytogenes in skim, whole and cho- colate milk, and in whipping cream during incubation at $4,8,13,21$ and $35^{\circ} \mathrm{C}$. J Food Prot 50, 452-459

Ryser ET, Marth EH (1987a) Behavior of Listeria monocytogenes during the manufacture and ripening of Cheddar cheese. J Food Prot 50, 7-13

Ryser ET, Marth EH (1987b) Fate of Listeria monocytogenes during the manufacture and ripening of Camembert cheese. J Food Prot $50,372-378$

Ryser ET, Marth EH, Doyle MP (1985) Survival of Listeria monocytogenes during manufacture and storage of Cottage cheese. J Food Prot 48, 746-750

Snedecor GW, Cochran WG (1957) Méthodes statistiques. Association de coordination technique agricole, Paris

Walker SJ, Archer P, Banks JG (1990) Growth of Listeria monocytogenes at refrigeration temperatures. J Appl Bacteriol 68, 157-160

Wilkins PO, Bourgeois R, Murray RGE (1972) Psychrotrophic properties of Listeria monocytogenes. Can J Microbiol 18, 543-551

Yousef AE, Marth EH (1988) Behavior of Listeria monocytogenes during the manufacture and storage of Colby cheese. J Food Prot 51, 12-15 